\title{
Five-year follow-up of nivolumab treatment in Japanese patients with esophageal squamous-cell carcinoma (ATTRACTION-1/ ONO-4538-07)
}

\author{
Taroh Satoh $^{1}$. Ken Kato ${ }^{2}$. Takashi Ura ${ }^{3,4}$. Yasuo Hamamoto ${ }^{5}$ Takashi Kojima $^{6}$. Takahiro Tsushima ${ }^{7}$. \\ Shuichi Hironaka ${ }^{8,9} \cdot$ Hiroki Hara $^{10} \cdot$ Satoru $_{\text {Iwasa }}{ }^{2} \cdot$ Kei Muro $^{3} \cdot$ Hirofumi Yasui ${ }^{7} \cdot K^{2}$ Keiko Minashi ${ }^{8}$. \\ Kensei Yamaguchi ${ }^{10,11}$. Atsushi Ohtsu ${ }^{12} \cdot$ Yuichiro Doki $^{13} \cdot$ Yasuhiro Matsumura $^{14} \cdot$ Yuko Kitagawa $^{15}$
}

Received: 17 March 2021 / Accepted: 4 May 2021 / Published online: 16 May 2021

(c) The Author(s) 2021

\begin{abstract}
Background In the phase II ATTRACTION-1 study, nivolumab demonstrated a promising antitumor activity among Japanese patients with treatment-refractory advanced esophageal cancer. Here, we report the follow-up results of ATTRACTION-1 of $>5$ years.

Methods We enrolled patients with esophageal cancer that was refractory or intolerant to a standard chemotherapy. Then, nivolumab $(3 \mathrm{mg} / \mathrm{kg}$ ) was administered every 2 weeks. The primary endpoint was a centrally assessed objective response rate. Results Nivolumab was administered to 65 patients with esophageal squamous-cell carcinoma (ESCC). The centrally assessed objective response rate was $17.2 \%$. The overall survival rates at 3 and 5 years were $10.9 \%$ and $6.3 \%$, respectively. Three-year survivors tended to have more reduced target lesions. A total of $63.1 \%$ of the patients exhibited treatment-related adverse events, and no new safety signal was observed. Patients with select adverse events tended to have better overall survival than those without. No apparent chronological order was observed between the first response and the onset of select adverse events.

Conclusion Our follow-up analysis of more than 5 years is currently the longest and is the first to demonstrate that nivolumab has long-term efficacy and safety for advanced ESCC.
\end{abstract}

Keywords Nivolumab $\cdot$ Esophageal squamous cell carcinoma $\cdot$ Clinical trial $\cdot$ Immunotherapy

\section{Introduction}

Esophageal cancer is one of the most common malignancies, with over 500,000 cases of new diagnosis and death worldwide in 2020 [1]. Monotherapy of docetaxel or paclitaxel has been applied to patients with esophageal cancer that are refractory or intolerant to fluoropyrimidine plus platinum-based chemotherapy; however, these alternatives have generally poor long-term outcomes [2-4]. In addition, only few treatment options for second- or later-line therapy are available.

Immune checkpoint inhibitors, including nivolumab, have provided major breakthroughs in cancer therapy [5].

Taroh Satoh

taroh@cfs.med.osaka-u.ac.jp

Extended author information available on the last page of the article
In particular, long-term survival with durable responses is a remarkable feature of treatment with immune checkpoint inhibitors [6-12]. Nivolumab, which is an anti-PD-1 antibody, inhibits interaction between PD-1 and its ligands, such as PD-L1, thereby facilitating the antitumor activity of T lymphocyte [13]. In the Japanese phase II ATTRAC TION-1 study, nivolumab exhibited a promising antitumor activity in patients with esophageal cancer that was refractory or intolerant to a standard chemotherapy [14]. In the subsequent phase III ATTRACTION-3 study, nivolumab significantly improved the overall survival (OS) compared with chemotherapy as a second-line therapy for esophageal cancer [15]. Through these findings, nivolumab was approved in Japan in February 2020, for esophageal cancer that is refractory or intolerant to a standard chemotherapy, and nivolumab was further approved as a second-line treatment for esophageal cancer in South Korea, the United States, Taiwan, Brazil, and the European Union. 
We previously reported 2-year follow-up results of ATT RACTION-1 [16]. Here, we report the longest follow-up data of nivolumab monotherapy for esophageal cancer for at least 5 years in ATTRACTION-1.

\section{Methods}

\section{Study design and patients}

This study conformed to the Declaration of Helsinki and Good Clinical Practice guidelines. All patients provided written informed consent. The study protocol was approved by the institutional review board at each study site.

ATTRACTION-1 is an open-label, single-arm, multicenter, phase II study conducted in Japan. Detailed methods of ATTRACTION-1 were described elsewhere [14]. In brief, patients who had esophageal cancer in the cervical or thoracic esophagus and were refractory or intolerant to fluoropyrimidine-based, platinum-based, and taxane-based chemotherapy were enrolled between February 25, 2014 and November 14, 2014. Other inclusion criteria included the presence of at least one measurable lesion that was unsuitable for radical resection, an Eastern Cooperative Oncology Group performance status of 0 or 1, and adequate organ function.

\section{Procedures}

Patients intravenously received $3 \mathrm{mg} / \mathrm{kg}$ of nivolumab every 2 weeks in 6-week cycles until the disease progression or unacceptable toxicity. Computed tomography of the neck, chest, abdomen, and pelvis was performed every 6 weeks in the first 8 cycles and every 12 weeks thereafter. We assessed the antitumor activity according to the Response Evaluation Criteria in Solid Tumors version 1.1, which includes complete response (CR), partial response (PR), stable disease, progressive disease (PD), and not assessable. The Japan Clinical Oncology Group translation of the Common Terminology Criteria for Adverse Events version 4.0 was used for grading adverse events (AEs).

\section{Endpoints}

The primary endpoint was the centrally assessed objective response rate (ORR), which was defined as the proportion of patients who achieved CR or PR. The secondary endpoints were the OS, progression-free survival (PFS), maximum change in tumor burden, best overall response (BOR), and others.

\section{Statistical analysis}

After nivolumab was approved for esophageal cancer in Japan on February 21, 2020, administration of nivolumab as the study drug in ATTRACTION-1 discontinued and commercially available nivolumab was used. For efficacy endpoints, this study used the data as of March 5, 2020, which was the last day of efficacy evaluation, and safety analyses used the data as of June 12, 2020, which was the time of the final data cutoff. Patients who received at least one dose of nivolumab were assessed for safety endpoints, and those with at least one central assessment of tumor response were assessed for efficacy endpoints. OS and PFS were analyzed by the Kaplan-Meier method with estimated median values and their 95\% confidence intervals (CIs) calculated with normal approximation. In a post hoc analysis, OS according to experience of select AEs (Supplementary Table 1) for the whole study period was assessed by the Kaplan-Meier method. All statistical data were analyzed using SAS version 9.4 (SAS Institute Inc., Cary, NC, USA).

\section{Results}

\section{Patients and treatments}

ATTRACTION-1 enrolled 65 patients with esophageal squamous-cell carcinoma (ESCC). All 65 patients were assessed for safety; however, one patient had multiple primary tumor sites, thereby excluded from the efficacy analysis. Patient characteristics at baseline were described previously [14]. All patients had ESCC, and 21 (32.3\%) and 20 (30.8\%) patients received $\leq 2$ and $\geq 4$ prior systemic therapies, respectively.

At the approval date of nivolumab for ESCC in Japan, two patients were on the study treatment.

\section{Efficacy}

The centrally assessed ORR was $17.2 \%$, and three patients achieved CR (Supplementary Table 2). In patients achieving $\mathrm{CR}$ or $\mathrm{PR}$, the median time to initial response was 1.4 months, and the median duration of response was 11.2 months. Through investigator assessment, target lesions were found to be reduced in approximately half of the patients (Fig. 1).

The median OS was 10.8 months, and 3-, 4-, and 5-year OS rates were $10.9 \%, 7.8 \%$, and $6.3 \%$, respectively (Fig. 2a). Seven patients survived for more than 3 years, while four patients survived for more than 5 years. Meanwhile, the median PFS was 1.5 months, and the Kaplan-Meier curve 
of PFS reached a plateau with 3-, 4-, and 5-year PFS rates of $8.6 \%, 6.8 \%$, and $6.8 \%$, respectively (Fig. $2 b$ ).

\section{Long-term survivors}

Table 1 summarizes the BOR in patients stratified by survival periods. All of the three patients with $C R$ survived more than 5 years (Table 1). In addition, target lesions tended to reduce greatly in these long-term survivors (Fig. 1).

The characteristics of patients who survived more than 3 years are shown in Table 2. These 3 -year survivors included patients with stage IV disease, comprehensive pretreatment, and negative PD-L1 expression on tumors.

\section{Safety}

The AEs observed in $\geq 5 \%$ of the study population are shown in Supplementary Table 3. Of 65 patients, 56 (86.2\%) exhibited AEs of any grade, and 21 (32.3\%) had AEs of grade 3 or 4 . The most common AEs were diarrhea, pneumonia, and decreased appetite. Other notable AEs included abnormal hepatic function of any grade and of grade 3 or 4 found in $4(6.2 \%)$ and $2(3.1 \%)$ patients, respectively. Pneumonia was the only grade 3 or $4 \mathrm{AE}$ reported in $\geq 3$ patients. Additionally, 41 (63.1\%) patients showed treatment-related AEs, and 13 (20.0\%) had grade 3 or 4 . The most common treatment-related AEs were diarrhea and rash. Nonetheless, no treatment-related AEs led to death.

\section{Comparison of efficacy in patients with and without select AEs}

In Kaplan-Meier analysis, patients experiencing select AEs during the whole study period tended to have better OS than those without (Fig. 3a). Among 11 patients who achieved CR or PR, eight exhibited select AEs. One responder exhibited select AEs before response to nivolumab, whereas others exhibited select AEs at the similar timing of or after the response (Fig. 3b).

\section{Discussion}

This analysis in ATTRACTION-1 with a follow-up of $>5$ years demonstrated that nivolumab monotherapy has long-term efficacy and safety for patients with chemotherapy-refractory or -intolerant esophageal cancer. Seven and four patients survived for 3 and 5 years, respectively, while the ORR, median OS, and median PFS remained unchanged at 2 years of follow-up [16]. No new safety signals were identified.

The Kaplan-Meier curves of PFS almost plateaued at 1-year follow-up, and 4 out of 5 patients without disease progression at 2-year follow-up remained progression-free for more than 5 years after the first dose of nivolumab. Furthermore, a 5-year survivor had received no subsequent therapy for more than a year; thus, nivolumab could persistently suppress esophageal cancer progression. Five-year

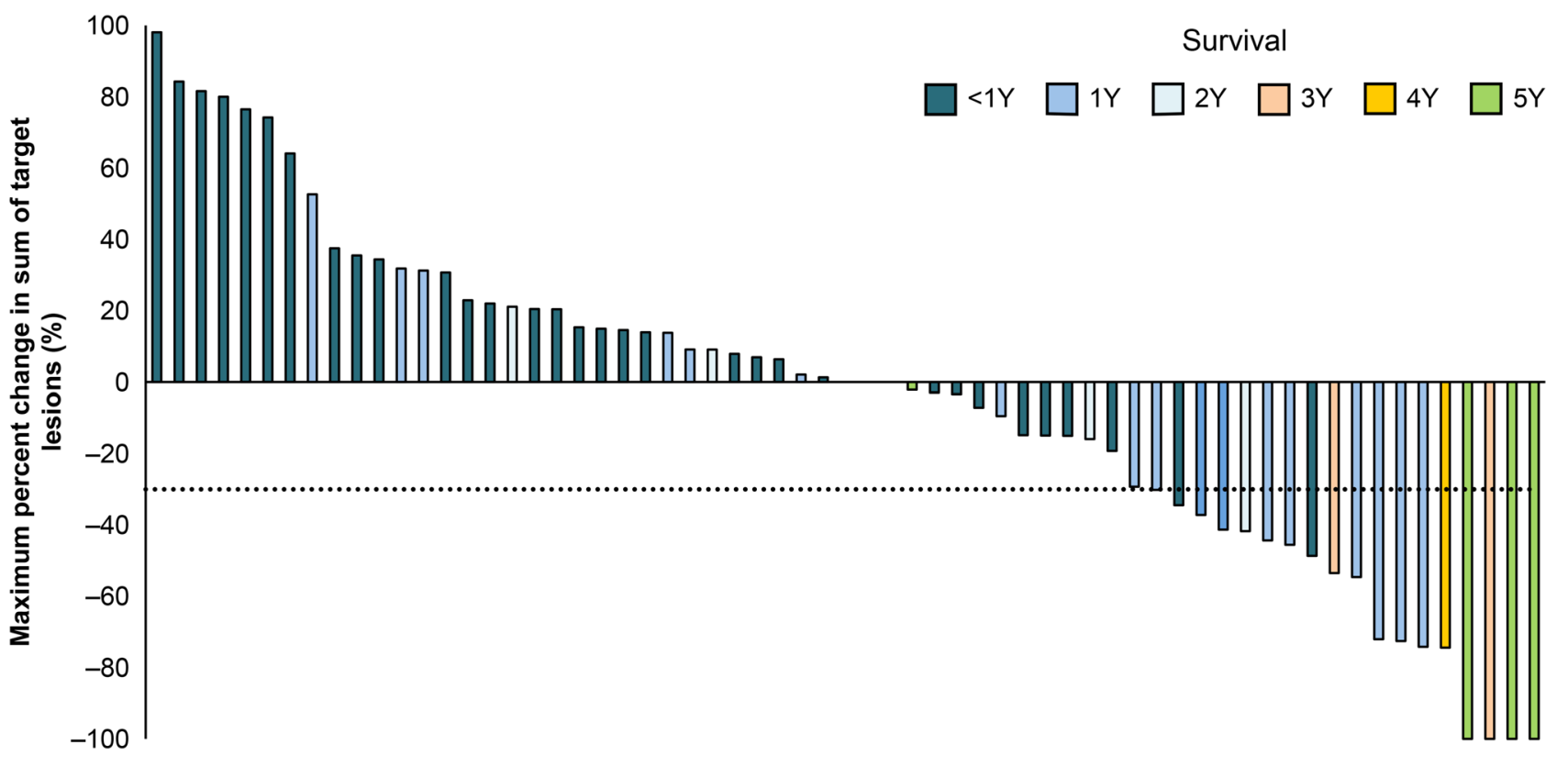

Fig. 1 Change of target lesion sizes. The waterfall plot highlights maximum percent change in the sum of target lesion size from baseline that were assessed by each investigator. Each color represents patients surviving for $<1$ (navy blue), 1 (blue), 2 (cyan), 3 (pink), 4 (orange), and 5 (green) years 
a

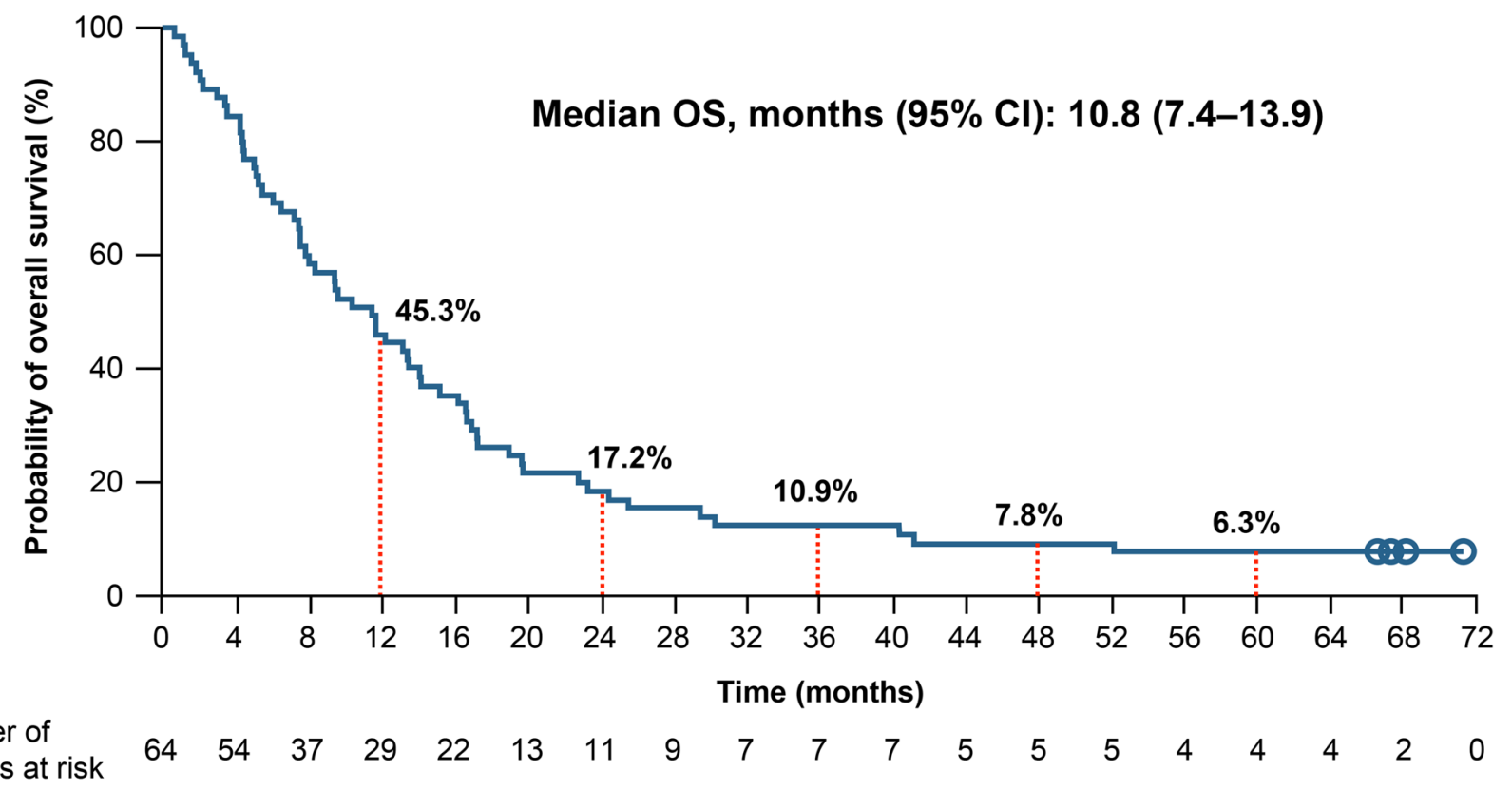

$\begin{array}{llllllllllllllllllllll}\begin{array}{l}\text { Number of } \\ \text { patients at risk }\end{array} & 64 & 54 & 37 & 29 & 22 & 13 & 11 & 9 & 7 & 7 & 7 & 5 & 5 & 5 & 4 & 4 & 4 & 2 & 0\end{array}$

b
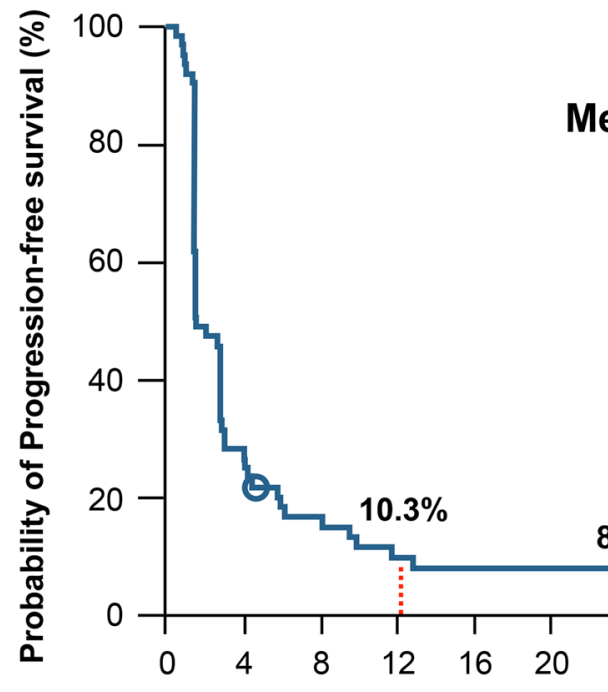

Median PFS, months (95\% CI): 1.5 (1.4-2.8)

Number of patients at risk

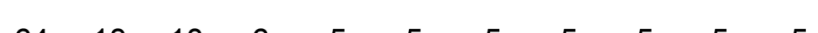

Time (months)

Fig. 2 Probability of survival. The Kaplan-Meier curves show the a overall survival (OS) and b progression-free survival (PFS). Circles represent censors. $\mathrm{CI}$, Confidence interval

survivors without disease progression were also observed in other tumors treated with nivolumab [10, 12].

This report has provided the longest and first 5-year efficacy and safety data of nivolumab for esophageal cancer. Here, two patients were treated with nivolumab for more than 5 years, suggesting that nivolumab monotherapy has long-term tolerability for esophageal cancer. The incidence of treatment-related AEs of grade 3 or 4 during 5-year treatment with nivolumab in patients with esophageal cancer was $20 \%$, which is consistent with those observed in patients with malignant melanoma, non-small 
Table 1 Best overall response of long-term survivors

\begin{tabular}{lclll}
\hline $\begin{array}{l}\text { Best overall response } \\
\text { (central review) }\end{array}$ & $\begin{array}{l}\text { All patients } \\
(N=64)\end{array}$ & $\begin{array}{l}\text { 2-year survivors } \\
(N=11)\end{array}$ & $\begin{array}{l}\text { 3-year survivors } \\
(N=7)\end{array}$ & $\begin{array}{l}\text { 5-year survivors } \\
(N=4)\end{array}$ \\
\hline Complete response & $3(4.7)$ & $3(27.3)$ & $3(42.9)$ & $3(75.0)$ \\
Partial response & $8(12.5)$ & $3(27.3)$ & $2(28.6)$ & 0 \\
Stable disease & $16(25.0)$ & $3(27.3)$ & $1(14.3)$ & 0 \\
Progressive disease & $29(45.3)$ & $1(9.1)$ & 0 & 0 \\
Not assessable & $8^{*}(12.5)$ & $1(9.1)$ & $1(14.3)$ & $1(25.0)$ \\
\hline
\end{tabular}

*Includes patients with no target lesions

Table 2 Characteristics of 3-year survivors

\begin{tabular}{|c|c|c|c|c|c|c|c|}
\hline & Patient 1 & Patient 2 & Patient 3 & Patient 4 & Patient 5 & Patient 6 & Patient 7 \\
\hline Sex & Male & Female & Male & Male & Female & Male & Male \\
\hline Age & 64 & 64 & 80 & 68 & 67 & 57 & 57 \\
\hline ECOG PS & 1 & 0 & 1 & 0 & 1 & 0 & 0 \\
\hline Alcohol consumption (habitual) & Former & Former & Former & Current & Current & Current & Former \\
\hline Smoking status & Former & Former & Former & Former & Former & Former & Former \\
\hline Prior radiation therapy & No & Yes & Yes & No & Yes & Yes & No \\
\hline Prior surgery & Yes & No & Yes & Yes & No & Yes & Yes \\
\hline Number of prior systemic therapy & 4 & 2 & 3 & 2 & 3 & 3 & 6 \\
\hline Disease stage & IIB & IIIC & IV & IIB & IA & IV & unknown \\
\hline Size of the target lesion $(\mathrm{mm})$ & 111 & 91.02 & 15.04 & 43.5 & 15.28 & 17.35 & 12.38 \\
\hline Tumor PD-L1 & $65 \%$ & ND & ND & $30 \%$ & $0 \%$ & $0 \%$ & $1 \%$ \\
\hline Metastases at diagnosis & Other LN & $\begin{array}{l}\text { Abdominal } \\
\text { LN, other } \\
\text { LN }\end{array}$ & $\begin{array}{c}\text { Cervical LN, } \\
\text { other LN }\end{array}$ & Other LN & No & $\begin{array}{l}\text { Other LN, } \\
\text { other organs }\end{array}$ & $\begin{array}{c}\text { Cervical LN, } \\
\text { Other LN }\end{array}$ \\
\hline Time of receiving nivolumab (months) & 70.8 & 67.6 & 55.4 & 46.9 & 47.2 & 10.3 & 29.2 \\
\hline OS (months) & 71.3 & 68.1 & 67.4 & 66.6 & 52.2 & 41.2 & 40.3 \\
\hline Best overall response (central review) & $\begin{array}{l}\text { Complete } \\
\text { response }\end{array}$ & $\begin{array}{l}\text { Complete } \\
\text { response }\end{array}$ & Not assessable & $\begin{array}{l}\text { Complete } \\
\text { response }\end{array}$ & $\begin{array}{l}\text { Partial } \\
\text { response }\end{array}$ & $\begin{array}{l}\text { Partial } \\
\text { response }\end{array}$ & $\begin{array}{l}\text { Stable } \\
\text { disease }\end{array}$ \\
\hline Subsequent therapy & Yes* & Yes* & No & No & No & Yes & Yes \\
\hline
\end{tabular}

*Commercially available nivolumab was used after approval

ECOG PS Eastern Cooperative Oncology Group Performance Status, $L N$ lymph node, $N D$ not determined, $O S$ overall survival

cell lung cancer, and renal cell carcinoma (16-23\%) [6-11]. The overall incidence of AEs is similar to that in the primary analysis of ATTRACTION-1 with a median follow-up of 10.8 months [14]; hence, most AEs tended to appear within a year of nivolumab treatment. The relatively early onset of AEs related to nivolumab treatment has also been observed in other cancers, including malignant melanoma, non-small cell lung cancer, and gastric cancer $[12,17,18]$. The current study observed that 5 and 2 patients manifested pneumonia as an AE of grade 3-4 and a treatment-related AE of grade 3-4, respectively, after the primary analysis. In addition, 5-year survivors with CR experienced select AEs 4-5 years after the initial dose of nivolumab. Therefore, relatively late-onset AEs should also be carefully monitored.

In this study, patients achieving objective responses accounted for most of the long-term survivors.
Additionally, patients exhibiting select AEs tended to survive longer than those without. Thus, even if the patients do not initially respond well to nivolumab, select AEs should be appropriately managed to expect for a longterm survival. Experience of select AEs could be a possible predictor of objective responses and long-term survival [19-21]. Consistent with our study, most patients achieving CR or PR experienced select AEs. However, some patients without select AEs also achieved an objective response. Furthermore, experience of select AEs and objective responses had no apparent chronological orders, as shown in head and neck cancer [22]. Some patients exhibited select AEs before an objective response, while others responded first and then exhibited select AEs. Thus, absence of select AEs should not be an indication to determine the discontinuation of nivolumab treatment. 
a

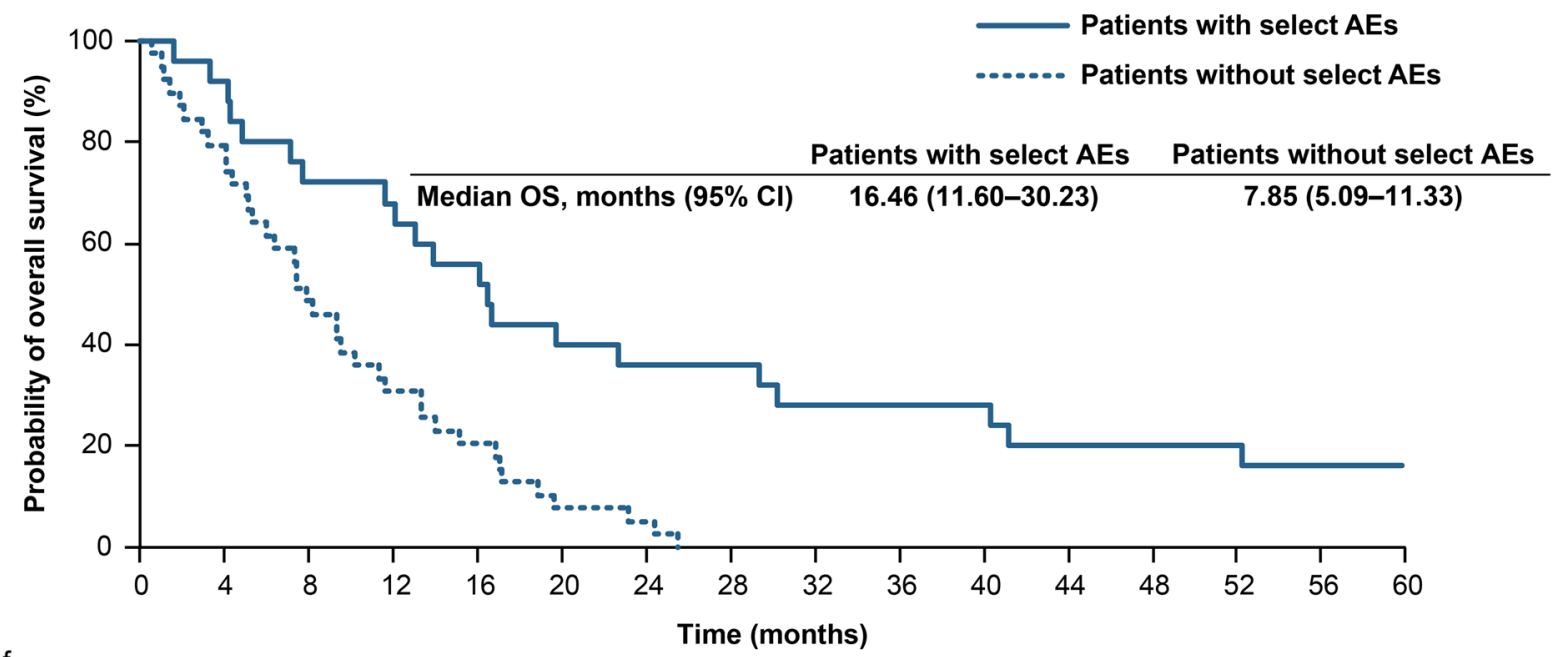

Number of patients at risk

$\begin{array}{lllllllllllllllll}\text { With select AEs } & 25 & 23 & 18 & 17 & 14 & 10 & 9 & 9 & 7 & 7 & 7 & 5 & 5 & 5 & 4 & 4 \\ \text { Without select AEs } & 39 & 31 & 19 & 12 & 8 & 3 & 2 & 0 & 0 & 0 & 0 & 0 & 0 & 0 & 0 & 0\end{array}$

b

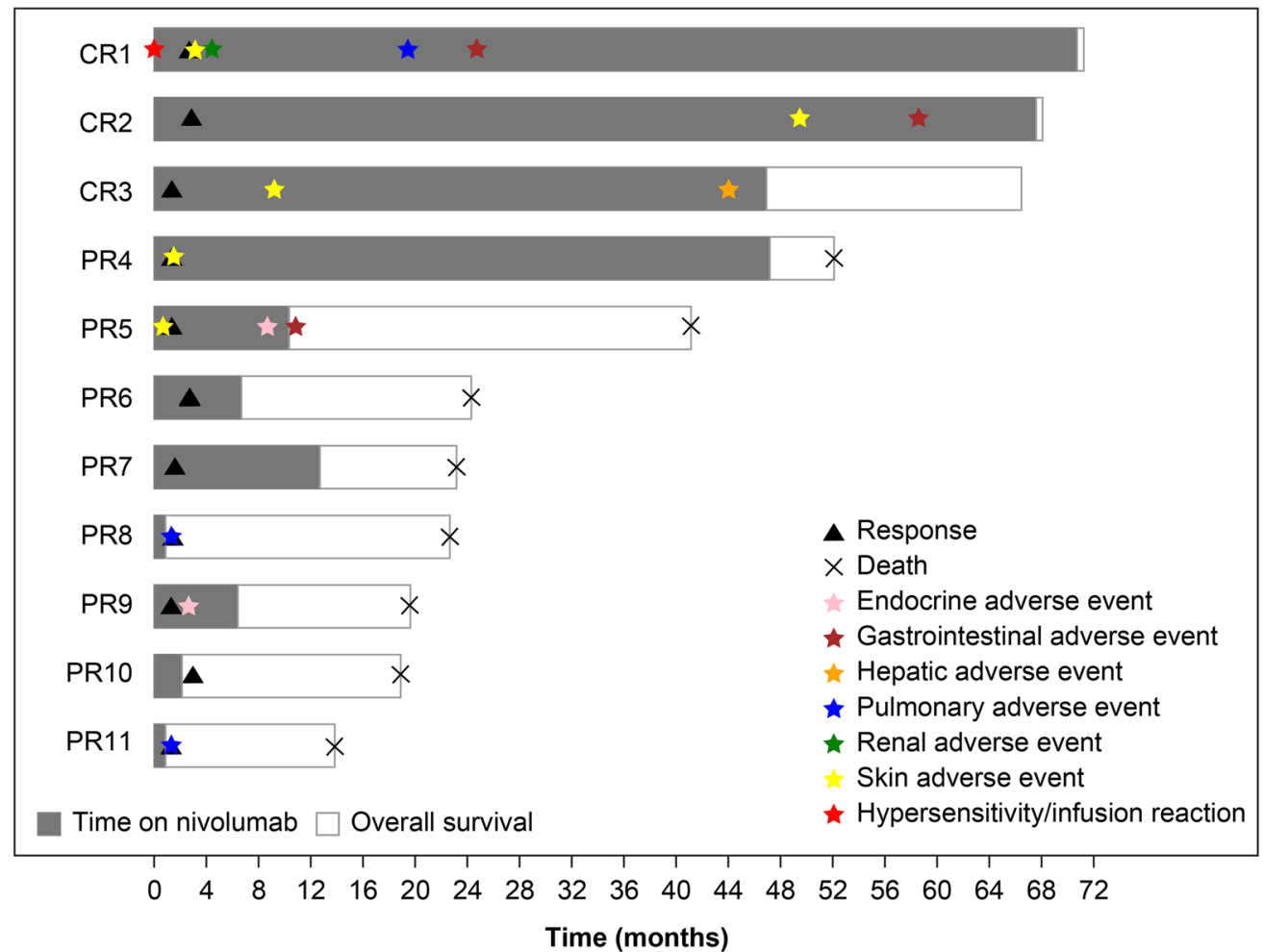

Fig. 3 Relationships between select AEs and overall survival or response. a The Kaplan-Meier curves shows the overall survival (OS) in patients with and without select adverse events (AEs) are shown. b The swimmer plot shows the timing of the response and the first onsets of each category of the treatment-related adverse events in patients with complete response (CR) or partial response (PR) in the nivolumab group 
We acknowledge some limitations in ATTRACTION-1. The single-arm study did not have any comparator groups. Only Japanese patients with ESCC were enrolled. Considering the small sample size, interpretations of subgroup analyses were limited. Long-term survivors may further be characterized in the large-scale phase III ATTRACTION-3 study.

\section{Conclusion}

This analysis represented the longest follow-up of patients with advanced ESCC treated with nivolumab. These patients survived longer through nivolumab treatment, and no new safety signals were identified during the 5-year follow-up.

Supplementary Information The online version contains supplementary material available at https://doi.org/10.1007/s10388-021-00850-0.

Acknowledgements We thank the patients and their families, and investigators and staff members participating in this study. Medical writing assistance with authors' directions was provided by Masatoshi Esaki, PhD, of Ono Pharmaceutical Co., Ltd, Osaka, Japan. This study was funded by Ono Pharmaceutical and Bristol-Myers Squibb, Princeton, NJ, USA.

\section{Declarations}

Ethical statement This study was conducted in accordance with the Declaration of Helsinki and Good Clinical Practice guidelines. All patients provided written informed consent. The study protocol was approved by the institutional review board at each study site.

Conflict of interest Taroh Satoh received research funding from Ono and Bristol-Myers Squibb during the conduct of the study; and grants from Ono, Chugai, Yakult Honsha, Eli Lilly, Bristol-Myers Squibb, MSD, Gilead Sciences, Parexel, Daiichi Sankyo, and Astellas; honoraria from Ono, Chugai, Yakult Honsha, Eli Lilly, BristolMyers Squibb, and Daiichi Sankyo; and travel fee from Ono outside the submitted work. Ken Kato received research funding from Ono and Bristol-Myers Squibb during the conduct of the study; and grants from Ono, MSD, AstraZeneca, Bayer, Chugai, BeiGene, and Shionogi; honoraria from Ono and Bristol-Myers Squibb outside the submitted work. Takashi Ura, Yasuo Hamamoto, Hirofumi Yasui, and Yuichiro Doki received research funding from Ono and Bristol-Myers Squibb during the conduct of the study. Takashi Kojima received research funding from Ono and Bristol-Myers Squibb during the conduct of the study; and research funding from MSD, Astellas Amgen BioPharma, Taiho, Chugai, and Shionogi; and honoraria from Ono, Bristol-Myers Squibb, MSD, Astellas, Merck, and Oncolys BioPharma outside the submitted work. Takahiro Tsushima received research funding from Ono and Bristol-Myers Squibb during the conduct of the study; and honorarium from Ono outside the submitted work. Shuichi Hironaka received research funding from Ono and Bristol-Myers Squibb during the conduct of the study; and honoraria from Bristol-Myers Squibb, Ono, Taiho, Daiichi Sankyo, Eli Lilly, Chugai, Nippon kayaku, Tsumura, Sanofi, Merck, and AstraZeneca outside the submitted work. Hiroki Hara received research funding from Ono and Bristol-Myers Squibb during the conduct of the study; and research funding from
Astellas, AstraZeneca, Bayer, BeiGene, Boehringer Ingelheim, Chugai, Daiichi Sankyo, Dainippon Sumitomo, Eisai, Elevar Therapeutics, GSK, Incyte, Merck Biopharma, MSD, Ono, Pfizer, and Taiho; consulting fees from Boehringer Ingelheim, Daiichi Sankyo, Dainippon Sumitomo, Eli Lilly, MSD, and Ono; and honoraria from Bayer, Bristol-Myers Squibb, Chugai, Daiichi Sankyo, Kyowa Hakko Kirin, Eli Lilly, Merck Biopharma, MSD, Ono, Sanofi, Taiho, Takeda, and Yakult outside the submitted work. Satoru Iwasa received research funding from Ono, Bristol-Myers Squibb, and Pfizer during the conduct of the study; and honoraria from Ono and Bristol-Myers Squibb outside the submitted work. Kei Muro received research funding from Ono and Bristol-Myers Squibb during the conduct of the study; and research funding from Solasia, Merck Serono, Daiichi Sankyo, Parexel International, Pfizer, MSD, Amgen, Ono, Sanofi, and Taiho; consulting fees from Amgen, Ono, and AstraZeneca; and honoraria from Ono, Sanofi, Taiho, Chugai, Takeda, Eli Lilly, Bristol-Myers Squibb, and Bayer outside the submitted work. Keiko Minashi received research funding from Ono and Bristol-Myers Squibb during the conduct of the study; and research funding from MSD, Mediscience Planning, Merck Biopharma, Astellas, Taiho, and Daiichi Sankyo outside the submitted work. Kensei Yamaguchi received research funding from Ono and Bristol-Myers Squibb during the conduct of the study; and grants from Taiho, Yakult Honsha, Sanofi, Ono, and Elli Lilly; and honoraria Taiho, Chugai, Bristol-Myers Squibb, Merck Serono, Takeda, Ono, and Elli Lilly outside the submitted work. Atsushi Ohtsu received research funding from Ono and Bristol-Myers Squibb during the conduct of the study; and honorarium from Bristol-Myers Squibb outside the submitted work. Yasuhiro Matsumura is an employee and a stockholder of Ono. Yuko Kitagawa received research funding from Ono and Bristol-Myers Squibb during the conduct of the study; and grants from Takeda, Chugai, Taiho, Yakult Honsha, Asahi Kasei, Otsuka, Ono, Tsumura, EA, Astellas, Toyama Chemical, Medicon, Kaken, Eisai, Otsuka, Teijin, Nihon, and Nippon Covidien; and honoraria from Chugai, Taiho, Asahi Kasei, Otsuka, Shionogi, and Nippon Covidien outside the submitted work.

Open Access This article is licensed under a Creative Commons Attribution 4.0 International License, which permits use, sharing, adaptation, distribution and reproduction in any medium or format, as long as you give appropriate credit to the original author(s) and the source, provide a link to the Creative Commons licence, and indicate if changes were made. The images or other third party material in this article are included in the article's Creative Commons licence, unless indicated otherwise in a credit line to the material. If material is not included in the article's Creative Commons licence and your intended use is not permitted by statutory regulation or exceeds the permitted use, you will need to obtain permission directly from the copyright holder. To view a copy of this licence, visit http://creativecommons.org/licenses/by/4.0/.

\section{References}

1. Sung H, Ferlay J, Siegel RL,Global cancer statistics, et al. GLOBOCAN estimates of incidence and mortality worldwide for 36 cancers in 185 countries. CA Cancer J Clin. 2021;71:209-49. https://doi.org/10.3322/caac.21660.

2. Shirakawa T, Kato K, Nagashima K, et al. A retrospective study of docetaxel or paclitaxel in patients with advanced or recurrent esophageal squamous cell carcinoma who previously received fluoropyrimidine- and platinum-based chemotherapy. Cancer Chemother Pharmacol. 2014;74:1207-15.

3. Mizota A, Shitara K, Kondo C, et al. A retrospective comparison of docetaxel and paclitaxel for patients with advanced or recurrent 
esophageal cancer who previously received platinum-based chemotherapy. Oncology. 2011;81:237-42.

4. Abraham P, Gricar J, Zhang Y, et al. Real-world treatment patterns and outcomes in patients receiving second-line therapy for advanced/metastatic esophageal squamous cell carcinoma. Adv Ther. 2020;37:3392-403.

5. Chen DS, Mellman I. Oncology meets immunology: the cancerimmunity cycle. Immunity. 2013;39:1-10.

6. Robert C, Long GV, Brady B, et al. Five-year outcomes with nivolumab in patients with wild-type BRAF advanced melanoma. J Clin Oncol. 2020;38:3937-46.

7. Gettinger S, Horn L, Jackman D, et al. Five-year follow-up of nivolumab in previously treated advanced non-small-cell lung cancer: results from the CA209-003 study. J Clin Oncol. 2018;36:1675-84.

8. Topalian SL, Hodi FS, Brahmer JR, et al. Five-year survival and correlates among patients with advanced melanoma, renal cell carcinoma, or non-small cell lung cancer treated with nivolumab. JAMA Oncol. 2019;5:1411-20.

9. Saka H, Nishio M, Hida T, et al. Five-year follow-up results from phase II studies of nivolumab in Japanese patients with previously treated advanced non-small cell lung cancer: pooled analysis of the ONO-4538-05 and ONO-4538-06 studies. Jpn J Clin Oncol. 2021;51:106-13.

10. Larkin J, Chiarion-Sileni V, Gonzalez R, et al. Five-year survival with combined nivolumab and ipilimumab in advanced melanoma. N Engl J Med. 2019;381:1535-46.

11. Kanda S, Ohe Y, Goto Y, et al. Five-year safety and efficacy data from a phase Ib study of nivolumab and chemotherapy in advanced non-small-cell lung cancer. Cancer Sci. 2020;111:1933-42.

12. Borghaei H, Gettinger S, Vokes EE, et al. Five-year outcomes from the randomized, phase III trials checkmate 017 and 057: nivolumab versus docetaxel in previously treated non-small-cell lung cancer. J Clin Oncol. 2021;39:723-33. https://doi.org/10. 1200/JCO.20.01605.

13. Brahmer JR, Drake CG, Wollner I, et al. Phase I study of singleagent anti-programmed death-1 (MDX-1106) in refractory solid tumors: safety, clinical activity, pharmacodynamics, and immunologic correlates. J Clin Oncol. 2010;28:3167-75.
14. Kudo T, Hamamoto Y, Kato K, et al. Nivolumab treatment for oesophageal squamous-cell carcinoma: an open-label, multicentre, phase 2 trial. Lancet Oncol. 2017;18:631-9.

15. Kato K, Cho BC, Takahashi M, et al. Nivolumab versus chemotherapy in patients with advanced oesophageal squamous cell carcinoma refractory or intolerant to previous chemotherapy (ATT RACTION-3): a multicentre, randomised, open-label, phase 3 trial. Lancet Oncol. 2019;20:1506-17.

16. Kato K, Doki Y, Ura T, et al. Long-term efficacy and predictive correlates of response to nivolumab in Japanese patients with esophageal cancer. Cancer Sci. 2020;111:1676-84.

17. Kiyohara $Y$, Uhara $H$, Ito $Y$, et al. Safety and efficacy of nivolumab in Japanese patients with malignant melanoma: an interim analysis of a postmarketing surveillance. J Dermatol. 2018;45:408-15.

18. Chen L-T, Satoh T, Ryu M-H, et al. A phase 3 study of nivolumab in previously treated advanced gastric or gastroesophageal junction cancer (ATTRACTION-2): 2-year update data. Gastric Cancer. 2020;23:510-9.

19. Masuda K, Shoji H, Nagashima K, et al. Correlation between immune-related adverse events and prognosis in patients with gastric cancer treated with nivolumab. BMC Cancer. 2019;19:1-10.

20. Haratani K, Hayashi H, Chiba Y, et al. Association of immunerelated adverse events with nivolumab efficacy in non-small cell lung cancer. JAMA Oncol. 2018;4:374-8.

21. Ishihara $\mathrm{H}$, Takagi $\mathrm{T}$, Kondo $\mathrm{T}$, et al. Association between immune-related adverse events and prognosis in patients with metastatic renal cell carcinoma treated with nivolumab. Urol Oncol Semin Orig Investig. 2019;37:355.e21-355.e29.

22. Yen CJ, Kiyota N, Hanai N, et al. Two-year follow-up of a randomized phase III clinical trial of nivolumab vs. the investigator's choice of therapy in the Asian population for recurrent or metastatic squamous cell carcinoma of the head and neck (CheckMate 141). Head Neck. 2020;42:2852-62.

Publisher's Note Springer Nature remains neutral with regard to jurisdictional claims in published maps and institutional affiliations.

\section{Authors and Affiliations}

\section{Taroh Satoh $^{1} \cdot$ Ken Kato ${ }^{2}$ 'Takashi Ura ${ }^{3,4}$. Yasuo Hamamoto ${ }^{5}$ Takashi Kojima ${ }^{6} \cdot$ Takahiro Tsushima $^{7}$. Shuichi Hironaka ${ }^{8,9} \cdot$ Hiroki Hara $^{10}$. Satoru Iwasa ${ }^{2} \cdot$ Kei Muro $^{3} \cdot$ Hirofumi Yasui $^{7} \cdot$ Keiko Minashi $^{8}$. Kensei Yamaguchi ${ }^{10,11} \cdot$ Atsushi Ohtsu $^{12} \cdot$ Yuichiro Doki $^{13} \cdot$ Yasuhiro Matsumura $^{14} \cdot$ Yuko Kitagawa $^{15}$}

1 Department of Frontier Science for Cancer and Chemotherapy, Osaka University Graduate School of Medicine, E21-19, 2-2, Yamadaoka, Suita, Osaka 565-0871, Japan

2 Department of Head and Neck, Esophageal Medical Oncology / Department of Gastrointestinal Medical Oncology, National Cancer Center Hospital, Tokyo, Japan

3 Department of Clinical Oncology, Aichi Cancer Center Hospital, Nagoya, Japan

4 Department of Clinical Oncology, National Hospital Organization Kyoto Medical Center, Kyoto, Japan

5 Keio Cancer Center, Keio University School of Medicine, Tokyo, Japan
6 Department of Gastroenterology and Gastrointestinal Oncology, National Cancer Center Hospital East, Kashiwa, Japan

7 Division of Gastrointestinal Oncology, Shizuoka Cancer Center, Shizuoka, Japan

8 Clinical Trial Promotion Department, Chiba Cancer Center, Chiba, Japan

9 Department of Medical Oncology and Hematology, Oita University Faculty of Medicine, Oita, Japan

10 Department of Gastroenterology, Saitama Cancer Center, Saitama, Japan

11 Department of Gastroenterological Chemotherapy, The Cancer Institute Hospital of Japanese Foundation for Cancer Research, Tokyo, Japan 
12 National Cancer Center Hospital East, Kashiwa, Japan

13 Department of Gastroenterological Surgery, Osaka University Graduate School of Medicine, Osaka, Japan

14 Department of Oncology, ONO Pharmaceutical Co., Ltd., Osaka, Japan
15 Department of Surgery, Keio University School of Medicine, Tokyo, Japan 